Document downloaded from:

http://hdl.handle.net/10251/166527

This paper must be cited as:

Carando, D.; Sevilla Peris, P. (2009). Spectra of weighted algebras of holomorphic functions. Mathematische Zeitschrift. 263:887-902. https://doi.org/10.1007/s00209-0080444-0

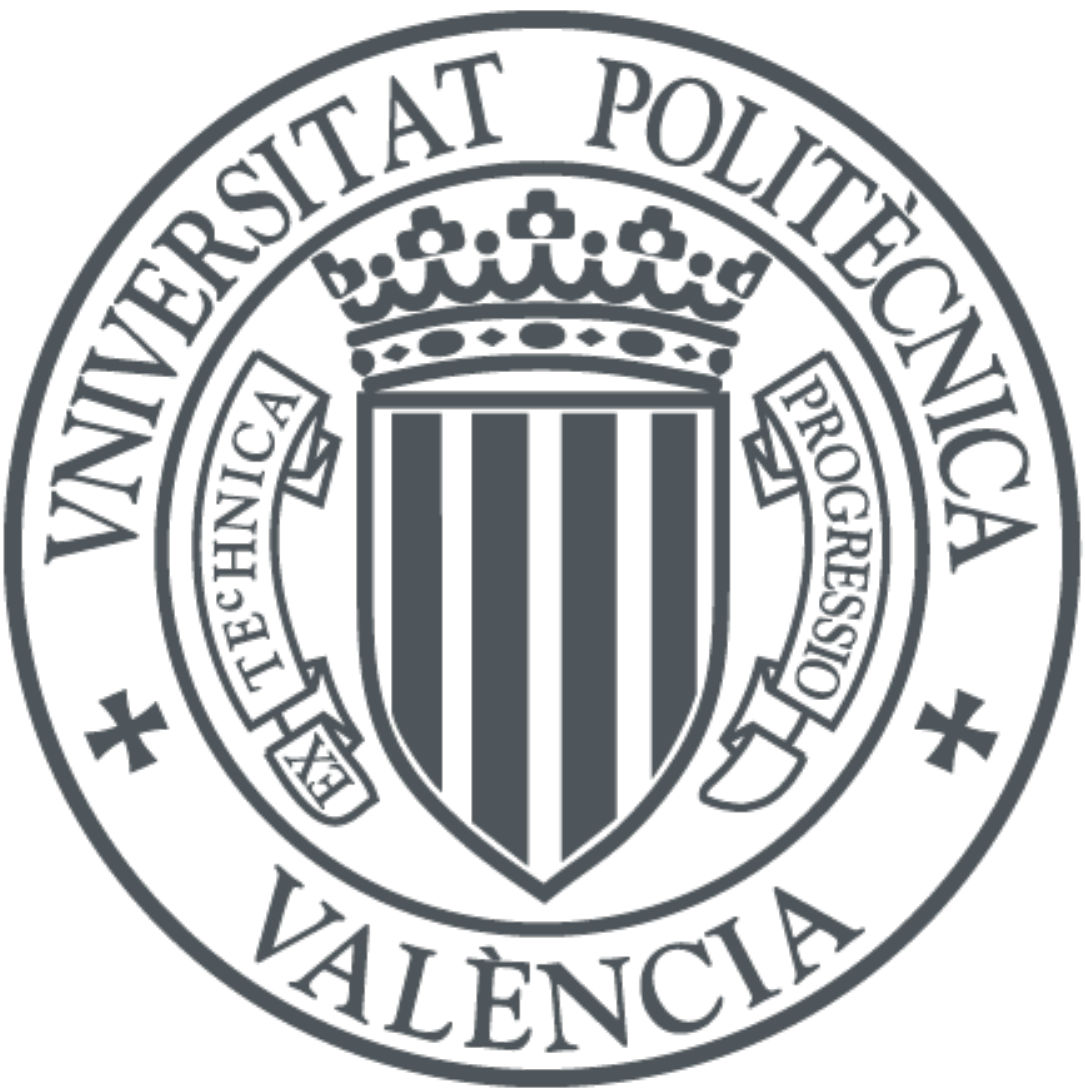

The final publication is available at

https://doi.org/10.1007/s00209-008-0444-0

Copyright Springer-Verlag

Additional Information 


\title{
Spectra of weighted algebras of holomorphic functions
}

\author{
Daniel Carando* \\ Departamento de Matemática, FCEyN, \\ Universidad de Buenos Aires, \\ Pabellón I - Ciudad Universitaria, 1428 \\ Buenos Aires, ARGENTINA. \\ dcarando@dm.uba.ar \\ Pablo Sevilla-Peris ${ }^{\dagger}$ \\ Departamento de Matemática Aplicada and IUMPA, \\ ETSMRE, \\ Universidad Politécnica de Valencia \\ Av. Blasco Ibáñez, 21 46010, \\ Valencia, Spain. \\ psevilla@mat.upv.es
}

May 13, 2021

\begin{abstract}
We consider weighted algebras of holomorphic functions on a Banach space. We determine conditions on a family of weights that assure that the corresponding weighted space is an algebra or has polynomial Schauder decompositions. We study the spectra of weighted algebras and endow them with an analytic structure. We also deal with composition operators and algebra homomorphisms, in particular to investigate how their induced mappings act on the analytic structure of the spectrum. Moreover, a Banach-Stone type question is addressed.
\end{abstract}

\section{Introduction}

This work deals with weighted spaces of holomorphic functions on a Banach space. If $X$ is a (finite or infinite dimensional) complex Banach space and $U \subseteq X$ open and balanced, by a weight we understand any continuous, bounded function $v: U \longrightarrow[0, \infty[$. Weighted spaces of holomorphic functions defined by countable families of weights were deeply studied by Bierstedt, Bonet and Galbis in 4 for open subsets of $\mathbb{C}^{n}$ (see also 5, 8, 9, 10, 12]). García, Maestre and Rueda defined and studied in 20 analogous spaces of functions defined on Banach spaces. We recall the definition of the weighted space

$$
H V(U)=\{f: U \rightarrow \mathbb{C} \text { holomorphic : }
$$

$$
\left.\|f\|_{v}=\sup _{x \in U} v(x)|f(x)|<\infty \text { all } v \in V\right\} .
$$

We endow $H V(U)$ with the Fréchet topology $\tau_{V}$ defined by the seminorms $\left(\|\|_{v}\right)_{v \in V}$. Since the family $V$ is countable, we can (and will throughout the article) assume it to be increasing.

One of the most studied topics on weighted spaces of holomorphic functions are the composition operators between them. These are defined in a very natural way; if $\varphi: \tilde{U} \rightarrow U$ is a holomorphic mapping and $V, W$ are two families of weights, the associated composition operator $C_{\varphi}: H V(U) \rightarrow H W(\tilde{U})$ is

* The first author was supported by PIP 5272, UBACyT X108, PICT 05-33042 and PICT 06-00587.

$\dagger$ The second author was supported by the MECD Project MTM2005-08210 
defined as $C_{\varphi}(f)=f \circ \varphi$. There are a number of papers on this topic, both in the finite dimensional and infinite dimensional setting [9, 10, 11, 12, 21, 22.

Our aim in this paper is to study the algebra structure of $H V(U)$ whenever it exists. We determine conditions on the family of weights $V$ that are equivalent to $H V(U)$ being an algebra, and present some examples. We also consider polynomial decompositions of weighted spaces of holomorphic functions. We show how the existence of a polynomial $\infty$-Schauder decomposition and the presence of an algebra structure are related. This lead us to the consideration of weights with some exponential decay.

Whenever $H V(X)$ is an algebra, we study the structure of its spectrum. For a symmetrically regular $X$ (see definitions in Section 3), we endow the spectrum of $H V(X)$ with a topology that makes it an analytic variety over $X^{* *}$, much in the spirit of Aron, Galindo, García and Maestre's work 3 for the space of holomorphic functions of bounded type $H_{b}(X)$. We show that any function $f \in H V(X)$ extends naturally to an analytic function defined on the spectrum and this extension can be seen to belong, in some sense, to $H V(X)$.

We also study algebra homomorphisms and composition operators between spaces $H V(X)$ and $H V(Y)$, for $V$ a family of exponential weights. Namely, we consider the algebra of holomorphic functions of zero exponential type. This class of functions has been widely studied in function theory in one or several variables since the 1930's [6, 7] and, even nowadays, its interest also arises in areas such as harmonic and Fourier analysis, operator theory and partial differential equations in complex domains. Given an algebra homorphism, we investigate how the mapping induced between the spectra acts on the corresponding analytic structures. We show how in this setting composition operators have a very different behaviour as that for holomorphic functions of bounded type 14. The results on algebra homomorphisms allow us to address a Banach-Stone type question. Some recent articles on this kind of problems are [14, 27. A survey on different types of Banach-Stone theorems can be found in [23]. This question can be seen as a kind of converse of the problem studied, for example, in [17, 25, 13, 15].

We now recall some definitions and fix some notation. We will denote duals by $X^{*}$ if $X$ is a Banach space and $E^{\prime}$ if $E$ is a Fréchet space.

Given a weight $v$, its associated weight is defined as

$$
\tilde{v}(x)=\frac{1}{\sup \left\{|f(x)|: f \in H v(U),\|f\|_{v} \leq 1\right\}}=\frac{1}{\left\|\delta_{x}\right\|_{(H v(U))^{\prime}}},
$$

where $\delta_{x}$ is the evaluation functional. It is a well known fact [5, Proposition 1.2], that $\|f\|_{v} \leq 1$ if and only if $\|f\|_{\tilde{v}} \leq 1$ (hence $H v(U)=H \tilde{v}(U)$ isometrically). We also have in [5, Proposition 1.2], that $v \leq \tilde{v}$. However, it is not always true that there exists a constant $C$ for which $\tilde{v} \leq C v$; the weights satisfying this kind of equivalence with their associated weights are called essential. A weight $v$ is called radial if $v(x)=v(\lambda x)$ for every $\lambda \in \mathbb{C}$ with $|\lambda|=1$ and norm-radial if $v\left(x_{1}\right)=v\left(x_{2}\right)$ whenever $\left\|x_{1}\right\|=\left\|x_{2}\right\|$.

A set $A \subseteq U$ is called $U$-bounded if it is bounded and $d(A, X \backslash U)>0$. Holomorphic functions of bounded type on $U$ are those that are bounded on $U$-bounded subsets. The space of all these functions is denoted by $H_{b}(U)$. By $H^{\infty}(U)$ we denote the space of holomorphic functions that are bounded in $U$. Following [20, Definition 1], we say that a countable family of weights $V$ satisfies Condition I if for every $U$-bounded $A$ there is $v \in V$ such that $\inf _{x \in A} v(x)>0$. If $V$ satisfies Condition I, then $H V(U) \subseteq H_{b}(U)$ and the topology $\tau_{V}$ is stronger than $\tau_{b}$ (topology of uniform convergence on the $U$-bounded sets).

Given a Banach space $X$, the space of continuous, $n$-homogeneous polynomials on $X$ is denoted by $\mathscr{P}\left({ }^{n} X\right)$. For a given family of weights $V$, we write $\mathscr{P} V\left({ }^{n} X\right)=\mathscr{P}\left({ }^{n} X\right) \cap H V(X)$.

A locally convex algebra will be an algebra $\mathscr{A}$ with a locally convex structure so that multiplication is continuous. The spectrum of $\mathscr{A}$ is the space of non-zero continuous multiplicative functionals. In the sequel, by "algebra" we will mean a locally convex algebra.

We denote the spectrum of $H_{b}(X)$ by $\mathfrak{M}_{b}(X)$. Whenever $H V(X)$ is an algebra, we will denote its spectrum by $\mathfrak{M} V(X)$.

\section{Weighted algebras of holomorphic functions}

Next proposition determines conditions on the weights that make $H V(X)$ an algebra. We thank our friend José Bonet for helping us fixing the proof, the final form of which is due to him. 
Proposition 1.1. Let $U$ be an open and balanced subset of $X$ and $V$ be a family of radial, bounded weights satisfying Condition I. Then $H V(U)$ is an algebra if and only if for every $v$ there exist $w \in V$ and $C>0$ so that

$$
v(x) \leq C \tilde{w}(x)^{2} \text { for all } x \in U .
$$

Proof. Let us begin by assuming that $H V(U)$ is an algebra. Given $v \in V$ there are $C>0$ and $w_{1}, w_{2}$ so that $\|f g\|_{v} \leq C\|f\|_{w_{1}}\|g\|_{w_{2}}$. Since $V$ is increasing, we can assume $w_{1}=w_{2}=w$. Let us fix $x_{0} \in U$, and choose $f \in H w(X)$ with $\|f\|_{w} \leq 1$ such that $f\left(x_{0}\right)=1 / \tilde{w}\left(x_{0}\right)$ (see [5, Proposition 1.2]). Taking the Cesàro means of $f$ (see [4, Section 1], or [20, Proposition 4]) we have a sequence $\left(h_{j}\right)_{j} \subseteq H V(U)$ such that $\left\|h_{j}\right\|_{w} \leq 1$ and $\left|h_{j}\left(x_{0}\right)\right| \longrightarrow 1 / \tilde{w}\left(x_{0}\right)$ as $j \rightarrow \infty$. We can assume that $h_{j}\left(x_{0}\right) \neq 0$ for $j$ large enough and we get

$$
v\left(x_{0}\right)=v\left(x_{0}\right)\left|h_{j}\left(x_{0}\right)^{2}\right| \frac{1}{\left|h_{j}\left(x_{0}\right)^{2}\right|} \leq\left\|h_{j}^{2}\right\|_{v} \frac{1}{\left|h_{j}\left(x_{0}\right)^{2}\right|}
$$

$$
\leq C\left\|h_{j}\right\|_{w}^{2} \frac{1}{\left|h_{j}\left(x_{0}\right)\right|^{2}} \leq C \frac{1}{\left|h_{j}\left(x_{0}\right)\right|^{2}} .
$$

Letting $j \rightarrow \infty$ we finally obtain (11). Conversely, if (1) holds, the fact that $\|f\|_{w}=\|f\|_{\tilde{w}}$ for every $f$ easily gives that $H V(U)$ is an algebra.

The problem of establishing if a weighted space of functions is an algebra was considered by L. Oubbi in [26] for weighted spaces of continuous functions. In that setting, $C V(X)$ is an algebra if and only if for every $v \in V$ there are $C>0$ and $w \in V$ so that, for every $x \in X$

$$
v(x) \leq C w(x)^{2} .
$$

Let us note that for holomorphic functions, since $w \leq \tilde{w}$, if $(2)$ holds then $H V(U)$ is an algebra. On the other hand, if the family $V$ consists of essential weights, then $H V(U)$ is an algebra if and only if (2) holds.

Examples of families generating algebras can be constructed by taking a weight $v$ and considering the family $V=\left\{v^{1 / n}\right\}_{n=1}^{\infty}$. Since in the sequel we will want that these families satisfy Condition I, we have to impose $v$ to be strictly positive.

Not every weighted algebra can be constructed with " $1 / n$ " powers of a strictly positive weight. In [20, Example 14], a family of weights $W=\left\{w_{n}\right\}_{n}$ satisfying Condition I so that $H_{b}(U)=H W(U)$ is defined. If $U_{n}$ is a fundamental system of $U$-bounded sets, each $w_{n}$ is defined to be 1 on $U_{n}$ and 0 outside $U_{n+1}$ and such that $0 \leq w_{n} \leq 1$. Let us see that there is no positive weight $v$ such that $H_{b}(U)=H V(U)$ (where $V$ is defined as before). We can view the identity $i d: H W(U) \longrightarrow H V(U)$ as a composition operator $i d=C_{i d_{U}}$; then by [22, Proposition 11] (see also [11, Proposition 4.1]) for each $n \in \mathbb{N}$ there exists $m$ so that $C_{i d_{U}}: H_{w_{m}}(U) \longrightarrow H_{v^{1 / n}}(U)$ is continuous. Then [21, Proposition 2.3] (see also [10, Proposition 2.5]) gives that $v(x)^{1 / n} \leq K \tilde{w}_{m}(x)$ for all $x$. Choose $x_{0} \notin U_{m+1}$ and we have $v\left(x_{0}\right)=0$, so $v$ is not strictly positive.

Now we present some concrete examples of weighted algebras.

Example 1.2. Let $v$ be the weight on $B_{X}$ given by $v(x)=(1-\|x\|)^{\beta}$ and let us define $V=\left\{v^{1 / n}\right\}_{n}$. Then, $H^{\infty}\left(B_{X}\right) \varsubsetneqq H V\left(B_{X}\right) \varsubsetneqq H_{b}\left(B_{X}\right)$.

The first inclusion and the second strict inclusion are clear. To see that the first one is also strict, we choose $x^{*} \in X^{*}$ and $x_{0} \in X$ so that $\left\|x^{*}\right\|=\left|x^{*}\left(x_{0}\right)\right|=\left\|x_{0}\right\|=1$ and $f(x)=\log \left(1-x^{*}(x)\right)$. Clearly $f$ is holomorphic and not bounded on the open unit ball $B_{X}$. On the other hand, there exists a constant $C>0$ for which

$$
(1-\|x\|)^{\beta}\left|\log \left(1-x^{*}(x)\right)\right| \leq(1-\|x\|)^{\beta} \log \left|1-x^{*}(x)\right|+C .
$$

Now, if $\left|1-x^{*}(x)\right|>1$, then $\log \left|1-x^{*}(x)\right| \leq 2$. If $\left|1-x^{*}(x)\right|<1$, then $\left|1-x^{*}(x)\right| \geq|1-| x^{*}(x)\|\geq 1-\| x \|$ and

$$
(1-\|x\|)^{\beta} \log \left|1-x^{*}(x)\right| \leq(1-\|x\|)^{\beta} \log (1-\|x\|) .
$$

Since the mapping $t \in] 0,1] \rightsquigarrow\left(t^{\beta} \log t\right)$ goes to 0 as $t$ does, we have $f \in H V\left(B_{X}\right) \backslash H^{\infty}\left(B_{X}\right)$. 
Example 1.3. Let $v$ be the weight on $X$ given by $v(x)=e^{-\|x\|}$ and $V=\left\{v^{1 / n}\right\}_{n}$. When $X=\mathbb{C}^{n}$, this weighted space $H V\left(\mathbb{C}^{n}\right)$ is the very well known algebra of entire functions of zero exponential type (see, for example, [6], [7]). We have $H^{\infty}(X) \varsubsetneqq H V(X) \varsubsetneqq H_{b}(X)$. To see that the second inclusion is strict, take $x^{*} \in X^{*}$ and define $f(x)=e^{x^{*}(x)^{2}}$. It is immediate that $f$ is a holomorphic function of bounded type that is not in $H V(X)$. On the other hand, $H V(X)$ cannot be $H^{\infty}(X)$.

We end this section by showing another example of a family that gives an algebra but is not given by $\left\{v^{1 / n}\right\}$. We thank our friend Manolo Maestre for providing us with it.

Example 1.4. Let us consider a positive, decreasing function $\eta$ defined on $X$ and define $v_{n}(x)=$ $\sqrt[n]{\log (n(1+\|x\|)) \eta(\|x\|)}$. This clearly satisfies that $v_{n}(x) \leq v_{2 n}(x)^{2}$ for all $x$ but there is no $v$ such that $v_{n}=v^{1 / n}$.

\section{Schauder decomposition and weighted algebras}

In this section, we consider two natural families of weights obtained from a decreasing continuous function $\eta:\left[0, \infty[\longrightarrow] 0, \infty\left[\right.\right.$ such that $\lim _{t \rightarrow \infty} t^{k} \eta(t)=0$ for every $k \in \mathbb{N}$. Let us define two different families of weights, $v_{n}(x)=\eta(\|x\|)^{1 / n}$ and $w_{n}(x)=\eta\left(\frac{\|x\|}{n}\right), n \in \mathbb{N}$. Our aim is to study some properties of the weighted spaces $H V(X)$ and $H W(X)$, where $V=\left\{v_{n}\right\}_{n}$ and $W=\left\{w_{n}\right\}_{n}$. From what has already been said in the previous section, $H V(X)$ is always an algebra. Note that $v_{1}(x)=w_{1}(x)=\eta(\|x\|)$. For simplicity, we will write $v=v_{1}$ and $w=w_{1}$.

Following standard notation the real function $\eta$ can be radially extended to a weight on $\mathbb{C}$ by $\eta(z)=$ $\eta(|z|)$ for $z \in \mathbb{C}$ and its associated weight is given by

$$
\tilde{\eta}(t)=\frac{1}{\sup \{|g(z)|: g \in H(\mathbb{C})|g| \leq 1 / \eta \text { on } \mathbb{C}\}} .
$$

The following proposition, showed to us by José Bonet, shows how the associated weights are related.

Proposition 2.1. Let $X$ be a Banach space and $v$ a weight defined by $v(x)=\eta(\|x\|)$ for $x \in X$. Then $\tilde{v}(x)=\tilde{\eta}(\|x\|)$ for all $x \in X$.

Proof. Let us fix $x \in X$ and choose $x^{*} \in X^{*}$ such that $\left\|x^{*}\right\|=1$ and $x^{*}(x)=\|x\|$. If $h \in H(\mathbb{C})$ is such that $|h| \leq 1 / \eta$ then, for any $y \in X$,

$$
\left|\left(h \circ x^{*}\right)(y)\right|=\left|h\left(x^{*}(y)\right)\right| \leq \frac{1}{\eta\left(x^{*}(y)\right)} \leq \frac{1}{\eta(\|y\|)}=\frac{1}{v(y)} .
$$

So we have $\left\|h \circ x^{*}\right\|_{v} \leq 1$ and hence

$$
\frac{1}{\tilde{\eta}(\|x\|)}=\sup \{|h(\|x\|)|: h \in H(\mathbb{C}),|h| \leq 1 / \eta\}
$$

$$
\leq \sup \left\{|f(x)|: f \in H_{v}(X),\|h\|_{v} \leq 1\right\}=\frac{1}{\tilde{v}(x)} .
$$

Let us suppose now that $\tilde{v}(x)<\tilde{\eta}(\|x\|)$ for some $x \neq 0$. Then there exists $f \in H(X)$ with $\|f\|_{v} \leq 1$ such that $|f(x)|>1 / \tilde{\eta}(\|x\|)$. Let us define now $g: \mathbb{C} \rightarrow \mathbb{C}$ by $g(\lambda)=f(\lambda x /\|x\|)$; clearly $g \in H(\mathbb{C})$ and $|g(\lambda)| \leq 1 / \eta(\lambda)$ for all $\lambda \in \mathbb{C}$. Therefore $|g(\|x\|)| \leq 1 / \tilde{\eta}(\|x\|)$, but this contradicts the fact that $g(\|x\|)=f(x)$. This gives that $\tilde{v}(x)=\tilde{\eta}(\|x\|)$ for every $x \neq 0$. Both $\tilde{v}$ and $\tilde{\eta}$ are continuous since $\eta$ is so, then we also have $\tilde{v}(0)=\tilde{\eta}(0)$

As an immediate consequence of this result we have that $v$ is essential if and only if $\eta$ is so. Also note:

Remark 2.2. Proceeding as in the previous Proposition it can be easily shown that $\tilde{w}_{n}(x)=\tilde{\eta}(\|x\| / n)$. 
Our family $W$ was already defined and studied in [20, Example 16]. By [20, Theorem 11], $\left(\mathscr{P} W\left({ }^{n} X\right)\right)_{n}$ is an $\mathscr{S}$-absolute, $\gamma$-complete decomposition of $H V(X)$ (see [18, Definition 3.32] and [24, Definition 3.1]). Let us see that, furthermore, it is an $\infty$-Schauder decomposition. Let us recall that a Schauder decomposition $\left(F_{n}\right)_{n}$ of a Fréchet space $F$ is an $R$-Schauder decomposition ([19, Theorem 1]), whenever, for any $\left(x_{n}\right)_{n}$ with $x_{n} \in F_{n}, \sum_{n} x_{n}$ converges in $F$ if and only if $\limsup _{n}\left\|x_{n}\right\|^{1 / n} \leq 1 / R$. It is well known [19, Lemma 6] that any $\infty$-Schauder decomposition is $\mathscr{S}$-absolute.

By [20, Example 16], $\mathscr{P} W\left({ }^{n} X\right)=\mathscr{P} w\left({ }^{n} X\right)$ topologically for every $n$. Since $\infty$-Schauder decompositions are sequences of Banach spaces, we will always consider $\mathscr{P} W\left({ }^{n} X\right)$ as a Banach space with the norm $\|\cdot\|_{w}$.

Proposition 2.3. $\left(\mathscr{P} W\left({ }^{n} X\right)\right)_{n}$ is an $\infty$-Schauder decomposition of $H W(X)$.

Proof. We want to show that $\sum_{m} P_{m}$ converges in $\tau_{W}$ if and only if $\lim _{m}\left\|P_{m}\right\|_{w}^{1 / m}=0$. Let us suppose first that $\sum_{m} P_{m}$ converges in $\tau_{W}$. Taking a sequence $\alpha_{m}=1$ for all $m$, since it is an $\mathscr{S}$-Schauder decomposition, $\left\|\sum_{m} P_{m}\right\|_{\alpha}=\sum_{m}\left\|P_{m}\right\|_{w}$ converges. Then, given any $R>0$, we can take $n>R$ and

$$
\begin{aligned}
\sup _{x \in X}\left|P_{m}(x)\right| \eta(\|x\|) R^{m} \leq \sup _{x \in X}\left|P_{m}(x)\right| \eta(\|x\|) n^{m} \\
\quad=\sup _{x \in X}\left|P_{m}(n x)\right| \eta(\|y\|)=\sup _{y \in X}\left|P_{m}(y)\right| \eta\left(\frac{\|x\|}{n}\right)=\left\|P_{m}\right\|_{w_{n}} .
\end{aligned}
$$

Hence $\sum_{m}\left(\sup _{x \in X}\left|P_{m}(x)\right| \eta(\|x\|)\right) R^{m}<\infty$ for all $R>0$ and this implies $\lim _{m}\left\|P_{m}\right\|_{w}^{1 / n}=0$.

Now, if $\lim _{m}\left\|P_{m}\right\|_{w}^{1 / n}=0$, then $\sum_{m}\left(\sup _{x \in X}\left|P_{m}(x)\right| \eta(\|x\|)\right) R^{m}<\infty$ for all $R>0$. Using (3), $\sum_{m}\left\|P_{m}\right\|_{w_{n}}$ converges for all $n$ and this completes the proof.

The space $H W(X)$ is not necessarily an algebra. We want to find now conditions on the weight that make $H W(X)$ an algebra and to study how is $H W(X)$ related to $H V(X)$ in this case.

Proposition 2.4. $H W(X)$ is an algebra if and only if there exist $k>1$ and $C>0$ so that, for all $t$,

$$
\eta(k t) \leq C \tilde{\eta}(t)^{2} .
$$

If, furthermore, $\eta$ is essential, then $H W(X)$ is an algebra if and only if there exist $k>1$ and $C>0$ so that, for all $t$,

$$
\eta(k t) \leq C \eta(t)^{2} .
$$

In this case, $H W(X) \hookrightarrow H V(X)$ continuously and there exist positive constants $a, b$ and $\alpha$ so that $\eta(t) \leq a e^{-b t^{\alpha}}$ for all $t$.

Proof. By Proposition 1.1 and Proposition 2.1. if $H W(X)$ is an algebra, given $n=1$ there exist $C>0$ and $m$ such $\eta(t) \leq C \tilde{\eta}\left(\frac{t}{m}\right)^{2}$ for all $t$. This clearly implies (4). On the other hand, if (4) holds, given $n$ we can choose $m_{n}$ so that $m_{n}>k n$ and the fact that $\tilde{\eta}$ is decreasing (because $\eta$ is decreasing [10]), together with Proposition 1.1 and Remark 2.2 give that $H W(X)$ is an algebra.

Now, if $\eta$ is essential, condition (4) is equivalent to (5). In this case, $\eta(t) \leq C^{2^{n}-1} \eta\left(t / k^{n}\right)^{2^{n}}$ for all $t$ and $n$. Hence, given $m$ let us take $n$ such that $2^{n}>m$, then since $\eta$ is decreasing,

$$
\left(\frac{\eta(t)}{\eta(0)}\right)^{1 / m} \leq\left(\frac{\eta(t)}{\eta(0)}\right)^{1 / 2^{n}} \leq C^{1-1 / 2^{n}} \frac{\eta\left(t / k^{n}\right)}{\eta(0)^{1 / 2^{n}}}
$$

This gives $\eta(t)^{1 / m} \leq C^{1-1 / 2^{n}} \eta(0)^{1 / m-1 / 2^{n}} \eta\left(t / k^{n}\right)$. Therefore, $H W(X) \hookrightarrow H V(X)$ continuously.

Moreover, since $\eta(t) \rightarrow 0$ as $t \rightarrow \infty$, we can choose $r$ such that $C \eta(r)<1$. We have $\eta\left(k^{n} r\right) \leq$ $C^{2^{n}-1} \eta(r)^{2^{n}} \leq(C \eta(r))^{2^{n}}$ for all $n$. Now, for any $t>0$, let $n$ be such that $k^{n} r \leq t<k^{n+1} r$. We have

$$
\eta(t) \leq \eta\left(k^{n} r\right) \leq(C \eta(r))^{2^{n}} \leq(C \eta(r))^{\frac{1}{2}(t / r)^{\log _{k} 2}}
$$

which is bounded by $a e^{-b t^{\alpha}}$ for a proper choice of positive constants $a, b$ and $\alpha$. 
We have given conditions for $H W(X)$ to be an algebra. We also had that $\left(\mathscr{P} W\left({ }^{n} X\right)\right)_{n}$ is an $\infty$ Schauder decomposition of $H W(X)$. The fact that the polynomials form a Schauder decomposition of a space of holomorphic functions allows to derive some properties of the space of holomorphic functions from the properties of the spaces of homogeneous polynomials. Let us check when the polynomials are such a decomposition for $H V(X)$.

Let us first note that $\mathscr{P} V\left({ }^{n} X\right)=\mathscr{P} W\left({ }^{n} X\right)=\mathscr{P} v\left({ }^{n} X\right)$. We consider in $\mathscr{P} V\left({ }^{n} X\right)$ the norm $\|\cdot\|_{v}$. Then if $\left(\mathscr{P} V\left({ }^{n} X\right)\right)_{n}$ is an $\infty$-Schauder decomposition of $H V(X)$, by [19, Theorem 9], we get $H V(X)=$ $H W(X)$. Since we know that $H W(X)$ always admits such a decomposition, we have that the spaces of weighted polynomials form an $\infty$-Schauder decomposition of $H V(X)$ if and only if $H V(X)=H W(X)$. Moreover, we have

Proposition 2.5. If $\eta$ is essential, $\left(\mathscr{P} V\left({ }^{n} X\right)\right)_{n}$ is an $\infty$-Schauder decomposition of $H V(X)$ if and only if $H V(X)=H W(X)$.

In this case, there exist positive constants $a_{1}, a_{1}, b_{1}, b_{2}, \alpha_{1}$ and $\alpha_{2}$ such that $a_{1} e^{-b_{1} t^{\alpha_{1}}} \leq \eta(t) \leq$ $a_{2} e^{-b_{2} t^{\alpha}}$ for all $t$.

Proof. We only need to show the inequalities. If $H V(X)=H W(X)$, then $H W(X)$ is an algebra and the second inequality follows from Proposition 2.4. On the other hand, if $H V(X)=H W(X)$ there must exist $m \in \mathbb{N}$ and $C>0$ such that $w_{2}(x) \leq C v_{m}(x)$ for all $x \in X$. This means that

$$
\eta(t / 2) \leq C \eta(t)^{1 / m}
$$

for all $t$. Now we can proceed as in the last part of the proof of Proposition 2.4 to obtain the desired inequality.

Remark 2.6. There is a whole class of functions $\eta$ for which $H V(X)$ and $H W(X)$ coincide (and, then, they are algebras with a polynomial $\infty$-Schauder decomposition). Indeed, for any $b, \alpha>0$ we can define $\eta(t)=e^{-b t^{\alpha}}$. Since $\eta(t / n) \leq \eta(t)^{1 / n^{[\alpha]}}$ and $\eta(t)^{1 / n} \leq \eta\left(t / n^{1 /[\alpha]}\right)$, we have $H V(X)=H W(X)$ topologically.

On the other hand, Proposition 2.5 shows that any $\eta$ satisfying $H V(X)=H W(X)$ must be bounded below and above by functions of this type.

If we want $H V(X)$ to have a polynomial decomposition without being $H W(X)$, we must then weaken our expectation on the type of decomposition. The polynomials form an $\mathscr{S}$-Schauder, $\gamma$-complete decomposition of the weighted space of holomorphic functions whenever the family is formed by norm radial weights satisfying Conditions I and II' (see [20, Theorem 11]). Condition I was already introduced. We say that a family of weights satisfies Condition II' if for every $v$ in the family there exist $C>0, R>1$ and $w$ in the family so that $v(x) \leq C w(R x)$ for all $x$ [20, Proposition 8]. We can characterise Condition II' in terms of the function $\eta$. Note that this condition also imposes a relationship between $H V(X)$ and $H W(X)$

Proposition 2.7. The family $V$ satisfies Condition II' if and only if there exist $R>1$, and $\alpha, C>0$ so that, for all $t$,

$$
\eta(t)^{\alpha} \leq C \eta(R t)
$$

In this case, $H V(X) \hookrightarrow H W(X)$ continuously.

Proof. First of all, if $V$ satisfies Condition II', clearly given any $n$ there exist $m, R$ and $C$ so that $\eta(t)^{m / n} \leq C \eta(R t)$ for all $t$. On the other hand, if (6) holds, for any $n$ let us choose $m \geq \alpha n$. Then

$$
\frac{\eta(t)^{1 / n}}{\eta(0)^{1 /(\alpha n)}} \leq C\left(\frac{\eta(R t)}{\eta(0)}\right)^{1 /(\alpha n)} \leq C\left(\frac{\eta(R t)}{\eta(0)}\right)^{1 / m}
$$

and this gives that Condition II' holds.

Now, if $V$ satisfies Condition II' then for any given $n$ and $k$ we have $\eta(t / n) \leq \eta\left(R^{k} t / n\right)^{\alpha^{k}}$. Let $k$ be such that $R^{k}>n$ and $m$ such that $m-1 \leq 1 / \alpha^{k} \leq m$. The set $A=\{t: \eta(t) \geq 1\}$ is compact; let then $K=\sup _{A} \eta(t)^{\frac{1}{1 / \alpha^{k}}} / \eta(t)^{1 / m}$ and we have

$$
\eta(t / n) \leq \eta\left(\frac{R^{k}}{n} t\right)^{\alpha^{k}} \leq \eta(t)^{\frac{1}{1 / \alpha^{k}}} \leq K \eta(t)^{1 / m} .
$$

This completes the proof. 


\section{The spectrum}

Our aim is now to study the structure of the spectrum of $H V(X)$. This is well known for the space of holomorphic functions of bounded type, $H_{b}(X)$, when $X$ is symmetrically regular. A complex Banach space $X$ is said to be (symmetrically) regular if every continuous (symmetric) linear mapping $T: X \rightarrow X^{*}$ is weakly compact. Recall that $T$ is symmetric if $T x_{1}\left(x_{2}\right)=T x_{2}\left(x_{1}\right)$ for all $x_{1}, x_{2} \in X$. The first steps towards the description of the spectrum $\mathfrak{M}_{b}(X)$ of $H_{b}(X)$ were taken by Aron, Cole and Gamelin in their influential article [2]. In [3, Corollary 2.2] Aron, Galindo, García and Maestre gave $\mathfrak{M}_{b}(U)$ a structure of Riemann analytic manifold modeled on $X^{* *}$, for $U$ an open subset of $X$. For the case $U=X, \mathfrak{M}_{b}(X)$ can be viewed as the disjoint union of analytic copies of $X^{* *}$, these copies being the connected components of $\mathfrak{M}_{b}(X)$ ). In [18, Section 6.3], there is an elegant exposition of all these results. The study of the spectrum of the algebra of the space of holomorphic functions of bounded type was continued in [14. We continue in this trend by studying here $\mathfrak{M} V(X)$. In this section we present the analytic structure of $\mathfrak{M} V(X)$, in the spirit of the above mentioned results.

If $f$ is a holomorphic function defined on a Banach space $X$, we denote by $\bar{f}$ or $A B(f)$ the Aron-Berner extension of $f$ to $X^{* *}$ (see [1] and [18] for definitions and properties).

The copies of $X^{* *}$ are constructed in the following way: given an element $\phi$ in the spectrum, we lay a copy of $X^{* *}$ around $\phi$ considering, for each $z \in X^{* *}$, the homomorphism that on $f \in H V(X)$ takes the value $\phi(x \in X \rightsquigarrow \bar{f}(x+z))$. If we let $z$ move in $X^{* *}$, we obtain a subset of the spectrum that is isomorphic to $X^{* *}$. But this works only if $\phi$ can act on the function $x \in X \rightsquigarrow \bar{f}(x+z)$, that is, if this function belongs to $H V(X)$.

Lemma 3.1. Let $V$ be a family of weights satisfying Conditions I and II' such that every $v$ is decreasing and norm radial; then the mapping $H V(X) \longrightarrow H V(X)$ given by $f \rightsquigarrow f(\cdot+x)$ is well defined and continuous for every fixed $x \in X$.

Proof. The mapping in the statement can be viewed as a composition operator $C_{\varphi_{x}}$, where $\varphi_{x}: X \longrightarrow X$ is given by $\varphi_{x}(y)=x+y$. We use [22, Proposition 11] (see also [11, Proposition 4.1]) to see that it is continuous.

Since $V$ satisfies Condition II', given $v \in V$, we can take $R>1$ and $w_{1}$ so that $v(y) \leq w_{1}(R y)$ for all $y$. Hence, if $\|y\|>\frac{1}{R-1}\|x\|$, then $\|x+y\| \leq R\|y\|$ and $v(y) \leq w_{1}(R y) \leq w_{1}(x+y)$. Let now $w_{2}$ be so that $\inf _{\|y\| \leq \frac{1}{R-1}\|x\|} w_{2}(y)=c_{1}>0$; then,

$$
\sup _{\|y\| \leq \frac{1}{R-1}\|x\|} \frac{v(y)}{w_{2}(y+x)}<\infty .
$$

Choosing $w \geq \max \left(w_{1}, w_{2}\right)$ we finally obtain for some $K>0$,

$$
\sup _{y \in X} v(y)|f(x+y)| \leq \sup _{y \in X} \frac{v(y)}{w(x+y)} \sup _{y \in X} w(x+y)|f(x+y)| \leq K\|f\|_{w} .
$$

Since $v$ is a function of the norm, we can consider it defined both on $X$ and $X^{* *}$. Davie and Gamelin showed that the Aron-Berner extension is an isometry for polynomials with the usual norm. They first prove a more general version of this fact: if $z \in X^{* *}$, there is $\left(x_{\alpha}\right)_{\alpha} \subseteq X$ such that $\left\|x_{\alpha}\right\| \leq\|z\|$ for all $\alpha$ and $P\left(x_{\alpha}\right) \rightarrow \bar{P}(z)$ as $\alpha \rightarrow \infty$, for all polynomial $P$ on $X$ [16, Theorem 1]. By using their result we show now that the Aron Berner extension is also an isometry from $\mathscr{P} V\left({ }^{n} X\right)$ into $\mathscr{P} V\left({ }^{n} X^{* *}\right)$.

If $P \in \mathscr{P} v\left({ }^{n} X\right)$, clearly $\|P\|_{v} \leq\|\bar{P}\|_{v}$. Also we can choose $x_{\alpha}$ in such a way that $\left\|x_{\alpha}\right\| \leq\|z\|$ and

$$
v(z)|\bar{P}(z)| \leq \lim _{\alpha} v(z)\left|P\left(x_{\alpha}\right)\right| \leq \sup _{\alpha} v\left(x_{\alpha}\right)\left|P\left(x_{\alpha}\right)\right| \leq\|P\|_{v} .
$$

Therefore,

$$
\|P\|_{v}=\|\bar{P}\|_{v} .
$$

This implies that the Aron-Berner extension is a continuous homomorphism from $H V(X)$ in $H V\left(X^{* *}\right)$. This was showed to us by M. Maestre in a more general setting, namely if $v$ is continuous on straight lines or $w^{*}$-continuous on spheres. 
In what follows we consider a positive decreasing function $\eta$ such that there is $C>0$ with

$$
\eta(s) \eta(t) \leq C \eta(s+t)
$$

A simple example of such a function is $\eta(t)=e^{-t}$. We consider the family of weights $v_{n}(x)=\eta(\|x\|)^{1 / n}$, defined analogously on $X^{* *}$. The space $H V(X)$ is an algebra and, since (6) holds, $V$ satisfies Condition II' and the weighted polynomials form a Schauder decomposition of $H V(X)$. Also, by [20, Example 16] it contains all the homogeneous polynomials. In order to study $\mathfrak{M} V(X)$ we follow the notation and trends of [18, Section 6.3] for $\mathfrak{M}_{b}(X)$. We reproduce the construction for the sake of completeness.

Linear functionals belong to $H V(X)$, so we can define an onto mapping $\pi: \mathfrak{M} V(X) \longrightarrow X^{* *}$ by $\pi(\phi)=\left.\phi\right|_{X^{*}}$. Since the Aron-Berner extension is continuous, we can also define $\delta: X^{* *} \longrightarrow \mathfrak{M V}(X)$ given by $\delta(z)(f)=\bar{f}(z)$. For any given $f \in H V(X)$ there is an associated mapping $f^{\prime \prime}: \mathfrak{M} V(X) \longrightarrow \mathbb{C}$ defined by $f^{\prime \prime}(\phi)=\phi(f)$. The canonical embedding of $X$ into $X^{* *}$ is denoted by $J_{X}$.

For a fixed $z \in X^{* *}$, we consider $\tau_{z}(x)=J_{X} x+z$ for $x \in X$. Since there is no risk of confusion we also denote $\tau_{z}: H V(X) \longrightarrow H V(X)$ the mapping given by $\left(\tau_{z} f\right)(x)=\bar{f}\left(J_{X} x+z\right)=\bar{f}(\cdot+z)=\left(\bar{f} \circ \tau_{z}\right)(x)$. By Lemma 3.1 and the comments above on the Aron-Berner extension this mapping is well defined. As a consequence, we get $\phi \circ \tau_{z} \in \mathfrak{M} V(X)$ for every $\phi \in \mathfrak{M} V(X)$ and $z \in X^{* *}$. If $X$ is symmetrically regular, then $\tau_{z+w} f=\left(\tau_{z} \circ \tau_{w}\right) f$ for all $f \in H_{b}(X)$ [18, Lemma 6.28]. Since $V$ satisfies Condition I, we have $H V(X) \hookrightarrow H_{b}(X)$ and $\tau_{z+w}=\tau_{z} \circ \tau_{w}$ on $H V(X)$.

Also, if $x^{*} \in X^{*}$, we have $\tau_{z}\left(x^{*}\right)=z\left(x^{*}\right)+x^{*}$, and for $\phi \in \mathfrak{M} V(X), \pi\left(\phi \circ \tau_{z}\right)=\pi(\phi)+z$.

For any pair $\phi \in \mathfrak{M} V(X)$ and $\varepsilon>0$ we consider

$$
V_{\phi, \varepsilon}=\left\{\phi \circ \tau_{z}: z \in X^{* *},\|z\|<\varepsilon\right\}
$$

As in [18, Section 6.3] we obtain that $\mathscr{V}_{\phi}=\left\{V_{\phi, \varepsilon}\right\}_{\varepsilon>0}$ is a neighbourhood basis at $\phi$ for a Hausdorff topology on $\mathfrak{M} V(X)$ whenever $X$ is symmetrically regular. Moreover, $\pi(\phi)=\pi(\psi)$ if and only if $\phi=\psi$ or $V_{\phi, r} \cap V_{\psi, s}=\varnothing$ for all $r, s$; also $\mathfrak{M} V(X)$ is a Riemann domain over $X^{* *}$ whose connected components are "copies" of $X^{* *}$.

As we have already mentioned, Condition I assures that $H V(X) \hookrightarrow H_{b}(X)$. Moreover, all the polynomials belong to $H V(X)$, so the inclusion has dense range. Hence, we have a one to one identification $\mathfrak{M}_{b}(X) \hookrightarrow \mathfrak{M} V(X)$. We do not know whether or not they are equal. Note that they both consist of "copies" of $X^{* *}$.

We have the following commutative diagram

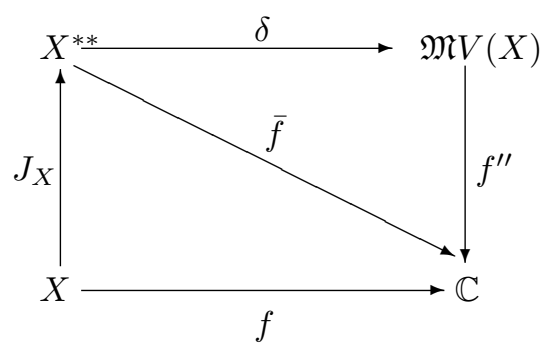

In the case of $H_{b}(X)$, the function $f^{\prime \prime}$ is holomorphic on $\mathfrak{M}_{b}(X)$ and is, in some sense, of bounded type. We show now that something analogous happens in our situation. By the Riemann domain structure of $\mathfrak{M} V(X)$, "holomorphic" means that $f^{\prime \prime} \circ\left(\left.\pi\right|_{V_{\phi, \infty}}\right)^{-1}$ is holomorphic on $X^{* *}$ for all $\phi \in \mathfrak{M} V(X)$, where $V_{\phi, \infty}=\bigcup_{\varepsilon>0} V_{\phi, \varepsilon}$.

Given a weight $v$ defined on $X$, we define the corresponding weighted norm for $n$-linear mappings:

$$
\|A\|_{v}=\sup _{x_{1}, \ldots, x_{n} \in X}\left|A\left(x_{1}, \ldots, x_{n}\right)\right| v\left(x_{1}\right) \cdots v\left(x_{n}\right) .
$$

If $P \in \mathscr{P}\left({ }^{n} X\right)$, we denote the associated symmetric $n$-linear mapping by $\check{P}$. For a symmetric $n$-linear mapping $A$, by $A\left(x^{k}, y^{n-k}\right)$ we mean the mapping $A$ acting $k$-times on $x$ and $(n-k)$ times on $y$. The following result follows by straightforward application of $(8)$ and the polarization formula [18]. 
Lemma 3.2. Let $\eta$ be a positive, decreasing function satisfying (8) and $v(x)=\eta(\|x\|)$. Then, for any $P \in \mathscr{P} v\left({ }^{n} X\right)$,

$$
\|\check{P}\|_{v} \leq \frac{C^{n}}{n !}\|P\|_{v}
$$

where $C$ is the constant in (8).

The following result is analogous to [18, Proposition 6.30] and follows the same steps.

Theorem 3.3. Let $X$ be symmetrically regular and $\eta$ be a positive, decreasing function satisfying (8). Let $V$ be defined by $v_{n}(x)=\eta(\|x\|)^{1 / n}$. Then, for every $f \in H V(X)$, the associated function $f^{\prime \prime}$ : $\mathfrak{M V}(X) \longrightarrow \mathbb{C}$ given by $f^{\prime \prime}(\phi)=\phi(f)$ is holomorphic.

Proof. For any $\phi \in \mathfrak{M} V(X)$ and $z \in X^{* *}$ we have

$$
\left(f^{\prime \prime} \circ\left(\left.\pi\right|_{V_{\phi, \infty}}\right)^{-1}\right)(\pi(\phi)+z)=f^{\prime \prime}\left(\phi \circ \tau_{z}\right)=\left(\phi \circ \tau_{z}\right)(f)=\phi\left(\tau_{z} f\right) .
$$

Hence we need to prove that the mapping $z \in X^{* *} \rightsquigarrow \phi\left(\tau_{z} f\right)=\phi\left(x \mapsto \bar{f}\left(J_{X} x+z\right)\right)$ is holomorphic.

Let us consider the polynomial expansion at zero: $f=\sum_{n} P_{n}$, where $P_{n} \in \mathscr{P}\left({ }^{n} X\right)$ for all $n$. What we need then is to show that the function $z \rightsquigarrow \phi\left(x \mapsto \sum_{n} \bar{P}_{n}(z)(x)\right)$ is holomorphic. To see it, this sum must converge for the topology $\tau_{V}$. We write $A_{n}=\check{P}_{n}$. For $z \in X^{* *}$ and $0 \leq k \leq n$ define $P_{n, k, z}: X \longrightarrow \mathbb{C}$ by $P_{n, k, z}(x)=\bar{A}_{n}\left(J_{X} x^{n-k}, z^{k}\right)$; this is clearly an $(n-k)$-homogeneous polynomial. Let us see that $P_{n, k, z}$ belongs to $\mathscr{P} V\left({ }^{n-k} X\right)$. For any $v \in V$, we set $w_{1}=v^{1 /(n-k)}$ and $w_{2}=v^{1 / k}$. Then, choosing $w \geq \max \left(w_{1}, w_{2}\right)$ we get

$$
\begin{aligned}
\left\|P_{n, k, z}\right\|_{v} & \leq \sup _{x \in X}\left|\bar{A}_{n}\left(J_{X} x^{n-k}, z^{k}\right)\right|\left(v(x)^{1 /(n-k)}\right)^{n-k} \frac{1}{v(z)}\left(v(z)^{1 / k}\right)^{k} \\
& \leq\left\|\bar{A}_{n}\right\|_{w} \frac{1}{v(z)}
\end{aligned}
$$

Now we apply Lemma 3.2 to obtain

$$
\left\|P_{n, k, z}\right\|_{v} \leq\left\|\bar{A}_{n}\right\|_{w} \frac{1}{v(z)} \leq \frac{1}{v(z)} \frac{C^{n}}{n !}\left\|\bar{P}_{n}\right\|_{w}=\frac{1}{v(z)} \frac{C^{n}}{n !}\left\|P_{n}\right\|_{w} .
$$

Proceeding as in [18, Section 6.3] we get a pointwise representation

$$
\left(\tau_{z} f\right)(x)=\bar{f}\left(J_{X} x+z\right)=\sum_{n=0}^{\infty} \bar{P}\left(J_{X} x+z\right)=\sum_{n=0}^{\infty}\left(\sum_{k=0}^{n}\left(\begin{array}{l}
n \\
k
\end{array}\right) P_{n, k, z}\right)(x) .
$$

This series converges in $\tau_{V}$; indeed if $v \in V$, inequality (9) gives

$$
\begin{aligned}
\sum_{n=0}^{\infty} \sum_{k=0}^{n}\left(\begin{array}{l}
n \\
k
\end{array}\right) \sup _{x \in X} v(x)\left|P_{n, k, z}(x)\right| & \\
& \leq \sum_{n=0}^{\infty} \sum_{k=0}^{n}\left(\begin{array}{l}
n \\
k
\end{array}\right) \frac{C^{n}}{n !} \frac{1}{v(z)}\left\|P_{n}\right\|_{w} \leq K \frac{1}{v(z)} \sum_{n=0}^{\infty}\left\|P_{n}\right\|_{w} .
\end{aligned}
$$

Since $\eta$ is strictly positive, so is $v$ and by [20, Lemma 10] the last series converges. Hence, for each $z \in X^{* *}$, the series $\sum_{n=0}^{\infty} \sum_{k=0}^{n}\left(\begin{array}{l}n \\ k\end{array}\right) P_{n, k, z}$ converges in $\tau_{V}$ to $\tau_{z} f$. Then we can write $\phi\left(\tau_{z} f\right)=\sum_{n=0}^{\infty} \sum_{k=0}^{n}\left(\begin{array}{l}n \\ k\end{array}\right) \phi\left(P_{n, k, z}\right)$.

Let us consider now the $k$-homogeneous polynomial $P_{n, k}: z \in X^{* *} \longrightarrow \phi\left(P_{n, k, z}\right)$ and see that it is continuous. We fix $w_{\phi} \in V$ such that $|\phi(h)| \leq M\|h\|_{w_{\phi}}$ for all $h \in H V(X)$. Note that $w_{\phi}$ coincides with $\eta(\|\cdot\|)^{1 / r}$ for some $r$. Let $z \in B_{X^{* *}}$, by $[9]$,

$$
\left|\phi\left(P_{n, k, z}\right)\right| \leq M\left\|P_{n, k, z}\right\|_{w_{\phi}} \leq M \frac{C^{n}}{n !}\left\|P_{n}\right\|_{w_{\phi}} \frac{1}{w_{\phi}(z)} \leq M \frac{1}{n !}\left\|P_{n}\right\|_{w_{\phi}} \frac{1}{\eta(1)^{1 / r}} .
$$

This means that $P_{n, k}$ is bounded and therefore $Q_{n}=\sum_{k=0}^{n}\left(\begin{array}{l}n \\ k\end{array}\right) \phi\left(P_{n, k, z}\right) \in \mathscr{P}\left({ }^{n} X^{* *}\right)$. Since $\phi\left(\tau_{z} f\right)=$ $\sum_{n=0}^{\infty} Q_{n}(z)$, we conclude that $\phi\left(\tau_{z} f\right)$ is a holomorphic function of $z$. 
We have shown that $f^{\prime \prime} \in H(\mathfrak{M} V(X))$. We can even get that in some sense it "belongs to $H V(\mathfrak{M} V(X))$ ". Let $\phi \in \mathfrak{M} V(X)$ and choose $w_{\phi}$ as before. For any $v \in V$, if we set $u \geq \max \left(w_{\phi}, v\right)$ and proceed as in the previous proof, we obtain

$$
\left|f^{\prime \prime}\left(\phi \circ \tau_{z}\right)\right| v(z) \leq M K \sum_{n=0}^{\infty}\left\|P_{n}\right\|_{u} .
$$

which is a finite constant by [20, Lemma 10]. Therefore, $f^{\prime \prime}$ belongs to $H V$ of each copy of $X^{* *}$ in the spectrum.

\section{Algebra homomorphisms between weighted algebras}

We now consider the weight given by $\eta(t)=e^{-t}$, and the associated family $V=\left\{v^{1 / n}\right\}_{n}$. This family obviously satisfies (8). Moreover, $V$ and $W$ coincide, and consequently the weighted spaces of polynomials are an $\infty$-Schauder decomposition of the algebra $H V(X)$ for any Banach space $X$.

We now study continuous algebra homomorphisms $A: H V(X) \longrightarrow H V(Y)$ and start by considering composition operators.

First, just a remark: if $f$ is a holomorphic function such that there exist $A, B>0$ with $|f(y)| \leq$ $A\|y\|+B$ for all $y \in Y$, then by the Cauchy inequalities $f$ is affine: there exist $y^{*} \in Y^{*}$ and $C>0$ so that $f(y)=y^{*}(y)+C$.

Lemma 4.1. Let $A: H V(X) \longrightarrow H V(Y)$ be an algebra homomorphism. Then Ax* is a degree 1 polynomial for all $x^{*} \in X^{*}$ (i.e. A maps linear forms on $X$ to affine forms on $Y$ ).

Proof. Since $A$ is continuous, given $n$, there exist $m$ and $C>0$ so that, for every $f \in H V(X)$

$$
\sup _{y \in Y}|A f(y)| e^{-\frac{\|y\|}{n}} \leq C \sup _{x \in X}|f(x)| e^{-\frac{\|x\|}{m}} .
$$

Let us take $x^{*} \in X^{*}$ and define $f(x)=\sum_{x=0}^{M} \frac{x^{*}(x)^{j}}{\left\|x^{*}\right\|^{j} m^{j} j !} \in H V(X)$. Since $A$ is an algebra homomorphism

$$
\begin{array}{r}
\sup _{y \in Y}\left|\sum_{j=0}^{M} \frac{\left(A x^{*}\right)(y)^{j}}{\left\|x^{*}\right\|^{j} m^{j} j !}\right| e^{-\frac{\|y\|}{n}} \leq C \sup _{x \in X}\left|\sum_{j=0}^{M} \frac{x^{*}(x)^{j}}{\left\|x^{*}\right\|^{j} m^{j} j !}\right| e^{-\frac{\|x\|}{m}} \\
\leq C \sup _{x \in X} \sum_{j=0}^{M} \frac{\left|x^{j}\right|}{m^{j} j !} e^{-\frac{\|x\|}{m}} \leq C \sup _{x \in X} e^{\frac{\|x\|}{m}} e^{-\frac{\|x\|}{m}}=C .
\end{array}
$$

This holds for every $M$; hence $\sup _{y \in Y}\left|e^{\frac{A x^{*}(y)}{\left\|x^{*}\right\| m}}\right| e^{-\frac{\|y\|}{n}} \leq C$. Then $\operatorname{Re}\left(\frac{A x^{*}}{\left\|x^{*}\right\|}(y)\right) \leq K_{1}\|y\|+K_{2}$ for all $y \in Y$. Also, if $|\lambda|=1$ we have $\operatorname{Re}\left(\lambda \frac{A x^{*}}{\left\|x^{*}\right\|}(y)\right)=\operatorname{Re}\left(A \frac{\lambda x^{*}}{\left\|x^{*}\right\|}(y)\right) \leq K_{1}\|y\|+K_{2}$. This gives $\left|A \frac{x^{*}}{\left\|x^{*}\right\|}(y)\right| \leq K_{1}\|y\|+K_{2}$ for all $y \in Y$. But this implies that $A \frac{x^{*}}{\left\|x^{*}\right\|}$ is affine on $y$; hence so is $A x^{*}$.

Corollary 4.2. If the composition operator $C_{\varphi}: H V(X) \longrightarrow H V(Y)$ is continuous, then $\varphi$ is affine.

Proof. By Lemma 4.1, $x^{*} \circ \varphi=C_{\varphi}\left(x^{*}\right)$ is affine. Since weakly affine mappings are affine, we obtain the conclusion.

It is clear that Lemma 4.1 and Corollary 4.2 are not valid for operators from $H_{b}(X)$ to $H_{b}(Y)$. Indeed, for any $\varphi \in H_{b}(Y, X)$, the composition operator $C_{\varphi}$ is well defined and continuous from $H_{b}(X)$ to $H_{b}(Y)$. In some cases, one may even obtain a non-affine bianalytic $\varphi$. Indeed, if $f$ is any entire function on $\mathbb{C}$, the Henon mapping $h: \mathbb{C}^{2} \rightarrow \mathbb{C}^{2}$ given by $h(z, u):=(f(z)-c u, z)$ is bianalytic and, of course, is not affine unless $f$ is. Henon-type mappings in infinite dimensional Banach spaces were used in [14, Theorem $35]$ to obtain homomorphisms with particular behaviour. See comments below, after Corollary 4.5 .

As an application of the previous results, we obtain a Banach-Stone type theorem for $H V$. 
Theorem 4.3. If $H V(X) \cong H V(Y)$ as topological algebras, then $X^{*} \cong Y^{*}$.

If moreover both $X$ and $Y$ are symmetrically regular or $X$ is regular, then $H V(X) \cong H V(Y)$ if and only if $X^{*} \cong Y^{*}$.

Proof. Let $A: H V(X) \longrightarrow H V(Y)$ be an isomorphism; by Lemma 4.1, $A x^{*}$ is affine for every $x^{*} \in X^{*}$. Let us define $S: X^{*} \longrightarrow Y^{*}$ by $S x^{*}=A x^{*}-A x^{*}\left(0_{Y}\right)$. Clearly, $S$ is linear and continuous. We consider also $\tilde{S}: Y^{*} \longrightarrow X^{*}$ given by $\tilde{S} y^{*}=A^{-1} y^{*}-A^{-1} y^{*}\left(0_{X}\right)$. Taking into account that $A x^{*}\left(0_{Y}\right)$ and $A^{-1} y_{\tilde{S}}^{*}\left(0_{X}\right)$ are constants and that constants are invariant for both $A$ and $A^{-1}$, it is easily seen than $S$ and $\tilde{S}$ are inverse one to each other. So $X^{*}$ and $Y^{*}$ are isomorphic.

If $X$ and $Y$ are symmetrically regular and $S: X^{*} \longrightarrow Y^{*}$ is an isomorphism, by [25, Theorem 4] the mapping $\hat{S}: \mathscr{P}\left({ }^{n} X\right) \longrightarrow \mathscr{P}\left({ }^{n} Y\right)$ given by $\hat{S}(P)=\bar{P} \circ S^{*} \circ J_{Y}$ is an isomorphism. Since $\mathscr{P}\left({ }^{n} X\right)$ and $\mathscr{P}\left({ }^{n} Y\right)$ coincide with $\mathscr{P} v\left({ }^{n} X\right)$ and $\mathscr{P} v\left({ }^{n} Y\right)$, we have that $\hat{S}$ is an isomorphism between the weighted spaces of polynomials. Since $v$ is decreasing, using (7) we have

$$
\begin{aligned}
& \sup _{y \in Y} v(y)|\hat{S}(P)(y)|=\|S\|^{n} \sup _{y \in Y} v(y)\left|\bar{P}\left(\frac{S^{*}\left(J_{Y}(y)\right)}{\|S\|}\right)\right| \\
&=\|S\|^{n} \sup _{y \in Y} v\left(\frac{S^{*}\left(J_{Y}(y)\right)}{\|S\|}\right)\left|\bar{P}\left(\frac{S^{*}\left(J_{Y}(y)\right)}{\|S\|}\right)\right| \leq\|S\|^{n}\|P\|_{v}
\end{aligned}
$$

Hence $\|\hat{S}(P)\|_{v} \leq\|S\|^{n}\|P\|_{v}$ and analogously for $\hat{S}^{-1}$. The fact that $\mathscr{P} v\left({ }^{n} X\right)$ and $\mathscr{P} v\left({ }^{n} Y\right)$ are respectively $\infty$-Schauder decompositions of $H V(X)$ and $H V(Y)$, [19, Theorem 1] and the multiplicative nature of the Aron-Berner extension give the conclusion.

If either $X$ or $Y$ are regular, we proceed analogously using [13, Theorem 1].

The spectrum of $H V(X)$ is formed by a number of copies of $X^{* *}$ and each one of them is a connected component of $\mathfrak{M} V(X)$. This can be viewed as if each copy of $X^{* *}$ were a "sheet" and all those "sheets" were laying one over the other in such a way that all the points in a vertical line are projected by $\pi$ on the same element of $X^{* *}$.

Every algebra homomorphism $A: H V(X) \longrightarrow H V(Y)$ induces a mapping $\theta_{A}: \mathfrak{M} V(Y) \longrightarrow \mathfrak{M} V(X)$ defined by $\theta_{A}(\phi)=\phi \circ A$. The sheets (copies of $Y^{* *}$ ) are the connected components of $\mathfrak{M} V(Y)$. By the analytic structure of $\mathfrak{M} V(Y), \theta_{A}$ is continuous if and only if $\theta_{A}$ maps sheets into sheets. We want to characterize the continuity of $\theta_{A}$. In order to keep things simple and readable we change slightly our notation. From now on the elements of the biduals will be denoted by $x^{* *}$ and $y^{* *}$. Also, we will identify $X^{* *}$ and $Y^{* *}$ with their images $\delta(X)$ and $\delta(Y)$ in the respective spectra.

Theorem 4.4. Let $X$ and $Y$ be symmetrically regular Banach spaces and $A: H V(X) \longrightarrow H V(Y)$ an algebra homomorphism. Then, the following are equivalent.

(i) There exist $\phi \in \mathfrak{M} V(X)$ and $T: Y^{* *} \longrightarrow X^{* *}$ affine and $w^{*}-w^{*}$-continuous so that $A f(y)=$ $\phi(\bar{f}(\cdot+T y))$ for all $y \in Y$.

(ii) $\theta_{A}$ maps sheets into sheets.

(iii) $\theta_{A}$ maps $Y^{* *}$ into a sheet.

In particular, $\theta_{A}$ is continuous if and only if it is continuous on $Y^{* *}$

Proof. Let us note first that $T: Y^{* *} \longrightarrow X^{* *}$ is affine and $w^{*}-w^{*}$-continuous if and only if there exist $R: X^{*} \longrightarrow Y^{*}$ linear and continuous and $x_{0}^{* *} \in X^{* *}$ so that $T\left(y^{* *}\right)=R^{\prime}\left(y^{* *}\right)+x_{0}^{* *}$.

We begin by assuming that $(i)$ holds. If $A$ has such a representation, let us see that then the AronBerner extension of $A f$ is of the form

$$
\overline{A f}\left(y^{* *}\right)=\phi\left(\bar{f}\left(\cdot+T y^{* *}\right)\right) .
$$

Indeed, let $h(z)=\phi(f(\cdot+z))=\phi(x \mapsto f(x+z))$ for $z \in X$. By [2, Theorem 6.12] its Aron-Berner extension is given by $\bar{h}\left(x^{* *}\right)=\phi\left(f\left(\cdot+x^{* *}\right)\right)=\phi\left(x \mapsto f\left(x+x^{* *}\right)\right)$. We define now $\tilde{h}\left(y^{* *}\right)=\phi\left(\bar{f}\left(\cdot+T y^{* *}\right)\right)$. Then

$$
\tilde{h}\left(y^{* *}\right)=(\bar{h} \circ T)\left(y^{* *}\right)=\bar{h}\left(R^{\prime}\left(y^{* *}\right)+x_{0}^{* *}\right)=\left(\tau_{x_{0}^{* *}}(\bar{h}) \circ R^{\prime}\right)\left(y^{* *}\right) .
$$

Since $\bar{h}$ is the Aron-Berner extension of a function, $\tau_{x_{0}^{* *}}(\bar{h})$ is the Aron-Berner extension of some other function (use, for example, [2, Theorem 6.12]). On the other hand, by [2, Lemma 9.1] the composition of 
an Aron-Berner extension with the transpose of a linear mapping is again the Aron-Berner extension of some function. Hence $\tilde{h}=\tau_{x_{0}^{* *}}(\bar{h}) \circ R^{\prime}$ is the Aron-Berner extension of a function; but $\tilde{h}$ coincides with Af on $X$, therefore $\tilde{h}=\overline{A f}$ and $\overline{10}$ holds.

Now, to see that $\theta_{A}$ maps sheets into sheets it is enough to find $S: Y^{* *} \longrightarrow X^{* *}$ such that $\theta_{A}(\psi \circ$ $\left.\tau_{y^{* *}}\right)=\left(\theta_{A} \psi\right) \circ \tau_{S y^{* *}}$. We define $S y^{* *}=T y^{* *}+x_{0}^{* *}$. First we have

$$
\begin{aligned}
& \theta_{A}\left(\psi \circ \tau_{y^{* *}}\right)(f)=\left(\psi \circ \tau_{y^{* *}}\right)(A f) \\
&=\psi\left[y \mapsto \overline{A f}\left(y+y^{* *}\right)\right]=\psi\left[y \mapsto \phi\left[x \mapsto \bar{f}\left(x+T\left(y+y^{* *}\right)\right)\right]\right] \\
&=\psi\left[y \mapsto \phi\left[x \mapsto \bar{f}\left(x+T y+S y^{* *}\right)\right]\right] .
\end{aligned}
$$

Let us call $g(x)=\bar{f}\left(x+S y^{* *}\right)$. As above, we can check that its Aron-Berner extension is $\bar{g}\left(x^{* *}\right)=$ $\bar{f}\left(x^{* *}+S y^{* *}\right)$. With this we obtain

$$
\begin{aligned}
& \left(\theta_{A} \psi \circ \tau_{S y^{* *}}\right)(f)=\theta_{A} \psi\left[x \mapsto \bar{f}\left(x+S y^{* *}\right)\right]=\psi(A g) \\
& =\psi[y \mapsto A g(y)]=\psi[y \mapsto \phi[x \mapsto \bar{g}(x+T y)]] \\
& \quad=\psi\left[y \mapsto \phi\left[x \mapsto \bar{f}\left(x+T y+S y^{* *}\right)\right]\right]
\end{aligned}
$$

and (ii) holds. Clearly, (ii) implies (iii).

Let us suppose that $\theta_{A}$ maps $Y^{* *}$ into a single sheet. Hence, $\theta_{A}\left(\delta_{y^{* *}}\right)=\theta\left(\delta_{0}\right) \circ \tau_{S y^{* *}}=\phi \circ \tau_{S y^{* *}}$ for some $S y^{* *}$ in $X^{* *}$. This means that $\delta_{y^{* *}}(A f)=\left(\phi \circ \tau_{S y^{* *}}\right)(f)$ for all $f$ and from this $\overline{A f}\left(y^{* *}\right)=\phi\left(\bar{f}\left(\cdot+S y^{* *}\right)\right)$. Let us see that $S$ is affine.

Let $x^{*} \in X^{*}$, then $A x^{*}$ is a degree one polynomial and so is $\overline{A x^{*}}$. Also,

$$
\begin{aligned}
\overline{A x^{*}}\left(y^{* *}\right) & =\phi\left[x \mapsto A B\left(x^{*}\right)\left(x+S y^{* *}\right)\right] \\
& =\phi\left[x \mapsto x^{*}(x)+S y^{* *}\left(x^{*}\right)\right]=\phi\left(x^{*}\right)+S\left(y^{* *}\right)\left(x^{*}\right) .
\end{aligned}
$$

This shows that $S$ is $w^{*}$ affine; hence $S$ is affine.

Let us finish by proving that $S$ is $w^{*}-w^{*}$-continuous. Indeed, let $\left(y_{\alpha}^{* *}\right)_{\alpha}$ be a net $w^{*}$-converging to $y^{* *}$. By Lemma 4.1 we have, for every $x^{*} \in X^{*}, A x^{*}=y_{x^{*}}^{*}+\lambda_{x^{*}}$. Then $\overline{A x^{*}}\left(y_{\alpha}^{* *}\right)=y_{\alpha}^{* *}\left(y_{x^{*}}^{*}\right)+\lambda_{x^{*}}$ and this converges to $y^{* *}\left(y_{x^{*}}^{*}\right)+\lambda_{x^{*}}=\overline{A x^{*}}\left(y^{* *}\right)$. Finally, $\lim _{\alpha} S\left(y_{\alpha}^{* *}\right)=\lim _{\alpha} \overline{A x^{*}}\left(y_{\alpha}^{* *}\right)-\phi\left(x^{*}\right)=$ $\overline{A x^{*}}\left(y^{* *}\right)-\phi\left(x^{*}\right)=S\left(y^{* *}\right)\left(x^{*}\right)$ and this completes the proof.

The previous theorem characterizes the homomorphisms $A$ for which $\theta_{A}$ maps $Y^{* *}$ into a sheet. A particular case is when $Y^{* *}$ is mapped precisely to $X^{* *}$. These are those for which $\phi=\delta_{T_{1}(0)}$ for some $T_{1}$. Then

$$
\overline{A f}\left(y^{* *}\right)=\delta_{T_{1}(0)}\left[x \mapsto \bar{f}\left(x+T y^{* *}\right)\right]=\bar{f}\left(T_{1}(0)+T y^{* *}\right)=\left(f \circ T_{2}\right)\left(y^{* *}\right) .
$$

Following 14, we say that $A: H V(X) \rightarrow H V(Y)$ is an $A B$-composition homomorphism if there exists $g: Y^{* *} \rightarrow X^{* *}$ such that $\overline{A(f)}\left(y^{* *}\right)=\bar{f}\left(g\left(y^{* *}\right)\right)$ for all $f \in H V(X)$ and all $y^{* *} \in Y^{* *}$. By the proof of the previous theorem, if $A$ is an $A B$-composition homomorphism, then $g$ must be affine. We can state the following:

Corollary 4.5. Let $X$ and $Y$ be symmetrically regular Banach spaces and $A: H V(X) \rightarrow H V(Y)$ an algebra homomorphism. Then $\theta_{A}\left(Y^{* *}\right) \subset X^{* *}$ if and only if $A$ is the AB-composition homomorphism associated to an affine mapping.

We feel that some important differences between the weighted algebras studied here and the algebra of holomorphic functions of bounded type are worthy to be stressed. By Theorem 4.4 and the comments following it, any $A B$-composition homomorphism induces a continuous $\theta_{A}$. In [14, examples are presented of composition homomorphisms inducing discontinuous $\theta_{A}$. Also, there are examples of homomorphisms for which the induced mapping $\theta_{A}$ is continuous on $Y^{* *}$ but is not continuous on the whole $\mathfrak{M}_{b}(Y)$ (i.e., splits some sheet other than $Y^{* *}$ into many sheets). Note that these homomorphisms are associated to composition operators given by polynomials of degree strictly greater than one, and would not work for $H V(X)$. 
A consequence of Corollary 4.5 is that, unless the spectrum of $H V(X)$ coincides with $X^{* *}$, there are homomorphisms on $H V(X)$ that are not $A B$-composition ones. Indeed, given any $\psi \in \mathfrak{M}_{b}(X)$, we can proceed as in the proof of Theorem 4.4 to obtain a homomorphism that maps $Y^{* *}$ into the sheet containing $\psi$. If $\psi$ does not belong to $X^{* *}$, the homomorphism thus obtained is not an $A B$-composition one.

The one to one identification $\mathfrak{M}_{b}(X) \hookrightarrow \mathfrak{M} V(X)$ leaves $X^{* *}$ invariant. If there exists a polynomial on $X$ that is not weakly sequentially continuous, then $\mathfrak{M}_{b}(X)$ properly contains $X^{* *}$ and then so does $\mathfrak{M} V(X)$. Therefore, if there are polynomials on $X$ that are not weakly sequentially continuous, then there are homomorphisms on $H V(X)$ other than $A B$-composition ones.

\section{Acknowledgements}

We would like to thank our friends: J. Bonet for all the help solving the difficulties with the associated weights, especially with the definite statement and proof of Proposition 1.1 and showing to us Proposition 2.1. M. Maestre for Example 1.4 and many discussions together with D. García that helped to improve the final shape of the article. We would also like to thank K. D. Bierstedt for useful remarks and comments.

Most of the work in this article was done while the second cited author was visiting the Department of Mathematics of the Universidad de Buenos Aires during the summer/winter of 2006 supported by grants GV-AEST06/092 and UPV-PAID-00-06. He wishes to thank all the people in and outside the Department that made that such a delightful time.

\section{References}

[1] R. M. Aron and P. D. Berner. A Hahn-Banach extension theorem for analytic mappings. Bull. Soc. Math. Fr., 106 (1978), 3-24.

[2] R. M. Aron, B. Cole, and T. W. Gamelin. Spectra of algebras of analytic functions on a Banach space. J. Reine Angew. Math., 415 (1991), 51-93.

[3] R. M. Aron, P. Galindo, D. García, and M. Maestre. Regularity and algebras of analytic functions in infinite dimensions. Trans. Amer. Math. Soc., 348(2) (1996), 543-559.

[4] K. D. Bierstedt, J. Bonet, and A. Galbis. Weighted spaces of holomorphic functions on balanced domains. Michigan Math., 40 (1993), 271-297.

[5] K. D. Bierstedt, J. Bonet, and J. Taskinen. Associated weights and spaces of holomorphic functions. Studia Math., 127(2) (1998), 137-168.

[6] R. P. Boas, Jr. Representations for entire functions of exponential type. Ann. of Math. (2), 39(2) (1938), 269-286.

[7] R. P. Boas, Jr. Entire functions. Academic Press Inc., New York, 1954.

[8] J. Bonet. Weighted spaces of holomorphic functions and operators between them. In U. d. S. Secretariado de Publicaciones, editor, Proceedings of the seminar of Mathematical Analysis (Univ. Málaga, Univ. Sevilla), pages 117-138, Sevilla, 2003.

[9] J. Bonet, P. Domański, and M. Lindström. Essential norm and weak compactness of composition operators on weighted spaces of analytic functions. Canad. Math. Bull., 42(2) (1999), 139-148.

[10] J. Bonet, P. Domański, M. Lindström, and J. Taskinen. Composition operators between wighted Banach spaces of analytic functions. J. Austral. Math. Soc. (Series A), 64 (1998) 101-118.

[11] J. Bonet and M. Friz. Weakly compact composition operators on locally convex spaces. Math. Nachr. 245 (2002), 26-44.

[12] J. Bonet, M. Friz, and E. Jordá. Composition operators between weighted inductive limits of sapces of holomorphic functions. Publ. Math. Debrecen, 67 no. 3-4 (2005), 333-348. 
[13] F. Cabello Sánchez, J. M. F. Castillo, and R. García. Polynomials on dual-isomorphic spaces. Ark. Mat., 38(1) (2000), 37-44.

[14] D. Carando, D. García, and M. Maestre. Homomorphisms and composition operators on algebras of analytic functions of bounded type. Adv. Math., 197(2) (2005), 607-629.

[15] D. Carando and S. Lassalle. $E^{\prime}$ and its relation with vector-valued functions on $E$. Ark. Mat., 42(2) (2004), 283-300.

[16] A. Davie and T. Gamelin. A theorem on polynomial-star approximation. Proc. Amer. Math. Soc., 106(2) (1989), 351-356.

[17] J. C. Díaz and S. Dineen. Polynomials on stable spaces. Ark. Mat., 36(1) (1998), 87-96.

[18] S. Dineen. Complex analysis on infinite dimensional spaces. Springer Verlag, London, 1999.

[19] P. Galindo, M. Maestre, and P. Rueda. Biduality in spaces of holomorphic functions. Math. Scand., 86(1) (2000), 5-16.

[20] D. García, M. Maestre, and P. Rueda. Weighted spaces of holomorphic functions on Banach spaces. Studia Math., 138(1) (2000), 1-24.

[21] D. García, M. Maestre, and P. Sevilla-Peris. Composition operators between weighted spaces of holomorphic functions on Banach spaces. Ann. Acad. Sci. Fenn. Math., 29 (2004), 81-98.

[22] D. García, M. Maestre, and P. Sevilla-Peris. Weakly compact composition operators between weighted spaces. Note Mat., 25(1) (2005/06), 205-220.

[23] M. I. Garrido and J. A. Jaramillo. Variations on the Banach-Stone theorem. Extracta Math., 17(3) (2002), 351-383. IV Course on Banach Spaces and Operators (Spanish) (Laredo, 2001).

[24] N. J. Kalton. Schauder decompositions in locally convex spaces. Proc. Cambridge Philos. Soc., 68 (1970), 377-392.

[25] S. Lassalle and I. Zalduendo. To what extent does the dual Banach space $E^{\prime}$ determine the polynomials over E? Ark. Mat., 38(2) (2000), 343-354.

[26] L. Oubbi. Weighted algebras of continuous functions. Results Math., 24(3-4) (1993), 298-307.

[27] D. M. Vieira. Theorems of Banach-Stone type for algebras of holomorphic functions on infinite dimensional spaces. Math. Proc. R. Ir. Acad., 106A(1):97-113, 2006. 